\title{
On the uniqueness of steady flow past a rotating cylinder with suction
}

\author{
By E. V. BULDAKOV, S. I. CHERNYSHENKO \\ AND A. I. RUBA N \\ Department of Mathematics, University of Manchester, Oxford Road, Manchester, \\ M13 9PL, UK
}

(Received 16 February 1999 and in revised form 1 December 1999)

The subject of this study is a steady two-dimensional incompressible flow past a rapidly rotating cylinder with suction. The rotation velocity is assumed to be large enough compared with the cross-flow velocity at infinity to ensure that there is no separation. High-Reynolds-number asymptotic analysis of incompressible NavierStokes equations is performed. Prandtl's classical approach of subdividing the flow field into two regions, the outer inviscid region and the boundary layer, was used earlier by Glauert (1957) for analysis of a similar flow without suction. Glauert found that the periodicity of the boundary layer allows the velocity circulation around the cylinder to be found uniquely. In the present study it is shown that the periodicity condition does not give a unique solution for suction velocity much greater than $1 / R e$. It is found that these non-unique solutions correspond to different exponentially small upstream vorticity levels, which cannot be distinguished from zero when considering terms of only a few powers in a large Reynolds number asymptotic expansion. Unique solutions are constructed for suction of order unity, $1 / R e$, and $1 / \sqrt{R e}$. In the last case an explicit analysis of the distribution of exponentially small vorticity outside the boundary layer was carried out.

\section{Introduction}

The problem of determining the velocity circulation around the closed boundary of a solid body in a two-dimensional fluid flow has been a focus of attention in fluid dynamics for almost a century. It is known that the Euler equations governing inviscid flow behaviour admit a family of solutions with circulation being an arbitrary parameter. For modelling non-separated flow around a body with a sharp trailing edge, say an airfoil, one may use the Kutta-Joukowskii condition for determining the unique magnitude of the circulation. This condition has in fact a viscous nature and eliminates the singular pressure gradient leading to separation from the sharp edge. However, it cannot be used for smooth bodies.

A simple example of non-separated flow past a smooth bluff body is the flow past a rapidly rotating circular cylinder. This flow was studied experimentally by Prandtl (see for example Prandtl \& Tietjens 1936). When the cylinder rotation is not fast enough, a large separation region is observed in the cylinder wake. However, this region shrinks and ultimately disappears when the cylinder wall velocity exceeds a certain value, being about twice the free-stream velocity.

The attached flow may be described by the well-known potential solution of the Euler equations with circulation depending upon the speed of the cylinder rotation. 
This circulation cannot be determined using the Euler equations only. Glauert (1957) was the first to calculate the circulation around the rotating cylinder. He used for this purpose Prandtl's equation for the boundary layer developing along the cylinder surface. By that time it had become clear (see Batchelor 1956; Squire 1956; Wood 1957) that a boundary layer with closed streamlines, like the one on the cylinder surface, does not always have a solution which is periodic in the circumferential direction. For the existence of the periodic solution an additional condition should be imposed on the external pressure distribution. Glauert (1957) demonstrated that this additional condition allows the circulation to be found as a function of the cylinder rotation speed. In his analysis Glauert used an asymptotic theory based on the assumption that the free-stream velocity is small compared with the velocity of the cylinder surface. As a result an explicit formula for the circulation was derived.

Later Nikolaev (1982) extended the theory to include the nonlinear effects in the boundary layer observed when the free-stream velocity becomes finite compared to the velocity of the cylinder surface. By means of numerical solution of the boundary layer equations subject to the periodicity condition he was able to study different flow regimes including those close to separation. In the present paper the influence of a suction through the cylinder surface upon the behaviour of the solution is studied.

It will be shown that even for very weak suction the condition of periodicity in the boundary layer is not sufficient to yield a unique solution.

This type of flow behaviour is well known. For example, in the case of zero freestream velocity and uniform suction the flow about a rotating cylinder is axisymmetric and an exact solution of the full Navier-Stokes equations is easily found (Rosenhead 1963). Written for the vorticity it has the form

$$
\omega=A\left(\frac{r_{0}}{r}\right)^{R},
$$

where $R=-r_{0} V_{w} / v$ is the Reynolds number based on the suction velocity, $r_{0}$ is the cylinder radius, $V_{w}<0$ is the suction velocity on the cylinder surface, and $A$ is the value of $\omega$ at the cylinder. If integrated in terms of the circumferential fluid velocity $v_{\theta}$ using

this solution gives

$$
\omega=\frac{1}{r} \frac{\partial}{\partial r}\left(r v_{\theta}\right)
$$

$$
v_{\theta}=\frac{\Gamma_{1}}{2 \pi r}+A\left(\frac{r_{0}}{r}\right)^{R-1} \quad(R \neq 2) .
$$

It follows that if $R<1$ then the only solution with zero velocity at infinity is $v_{\theta}=\Gamma_{1} /(2 \pi r), A=0$ with $\Gamma_{1}$ uniquely determined from the no-slip condition at the cylinder, but if $R>1$ then there is a one-parameter family of solutions with $A$ as an arbitrary parameter. Egorov (1986) solved the corresponding unsteady problem and showed that $A$ is determined by the initial conditions.

This result cannot be directly applied in the case of a small non-zero cross-flow velocity, because this limit is non-uniform. The reason is that the velocities caused by the suction and cylinder rotation tend to zero at infinity, and, hence, at sufficiently large distance from the cylinder they will be of the same order of magnitude as the cross-flow velocity, however small the latter is.

Our study in this paper is based on asymptotic analysis of the Navier-Stokes equations at large values of the Reynolds number. Section 2 is devoted to formulation 
of the problem and discussion of some properties of the expected solution. In $\S 3$ strong suction is considered and it is shown that Glauert's approach gives non-unique solutions while the use of a certain invariant condition described in $\S 2$ allows some of them to be excluded, or even unique solutions to be found for certain distributions of wall velocity. In $\S 4$ the solution is constructed for suction velocity of order $1 / \sqrt{R e}$ with results similar to those of $\S 3$, namely to determine the unique solution the invariant condition had to be used. In $\S 5$ the case of suction of order $1 / R e$ is analysed, a unique solution is obtained without use of the invariant condition, and the nature of the non-uniqueness of asymptotic solutions is revealed and discussed. This makes it possible to return to the case of suction of order $1 / \sqrt{R e}$ in $\S 6$, where an analysis of the exponentially small vorticity outside the boundary layer is performed, which allowed the unique asymptotics in this case to be found and clarified further the nature of the observed behaviour of the asymptotic expansion.

\section{Problem formulation and some properties of the solution}

The following boundary-value problem for the steady Navier-Stokes equations will be considered:

$$
\left.\begin{array}{c}
\boldsymbol{u} \cdot \nabla \boldsymbol{u}=-\nabla p+\frac{1}{R e} \nabla^{2} \boldsymbol{u}, \\
\nabla \cdot \boldsymbol{u}=0, \\
\left.\boldsymbol{u}\right|_{S}=\boldsymbol{u}_{w},\left.\quad \boldsymbol{u}\right|_{|x| \rightarrow \infty}=\boldsymbol{u}_{\infty},
\end{array}\right\}
$$

where $S$ is the cylinder surface. The velocity $\boldsymbol{u}_{w}$ at the wall has two components: the tangential velocity $u_{w}$ and the normal velocity, or suction velocity, $v_{w}$. In most cases we will assume that $u_{w}$ and $v_{w}$ are constants. The velocity $\boldsymbol{u}_{\infty}$ at infinity has only one non-zero component $u_{\infty}$, which will be assumed constant throughout the paper except in $\S 5$ where suction velocity of order $1 / R e$ is considered. In that section we will also consider the case of a shear flow at infinity, with a non-zero vorticity $\Omega_{\infty}$. In this case $u_{\infty}=-\Omega_{\infty} y$, where $y$ is the Cartesian coordinate directed perpendicular to $\boldsymbol{u}_{\infty}$. All the quantities are suitably non-dimensionalized so that the rotation velocity of the cylinder surface is of order unity.

For any closed contour we can introduce the velocity circulation $\Gamma$. If the solution of (2.1) is unique then $\Gamma$ may be considered as a function of $u_{w}$. When the highReynolds-number asymptotics is considered, far from the body the flow is potential, and $\Gamma$ does not depend on the particular contour enclosing the body. In that case it may be convenient to prescribe $\Gamma$ and seek the rotation velocity $u_{w}$ which ensures this value of $\Gamma$.

There are two properties of the solution of (2.1) which play an important role in the subsequent analysis. They can be described in terms of the vorticity flux density

$$
\boldsymbol{R}=\boldsymbol{u} \omega-\frac{1}{R e} \nabla \omega,
$$

where $\boldsymbol{u} \omega$ and $\nabla \omega /$ Re are vorticity fluxes due to convection and diffusion respectively. The Helmholtz equation for vorticity, which can be derived by taking curl of (2.1), has the form $\nabla \cdot \boldsymbol{R}=0$. Using the continuity equation it can also be written as

$$
\boldsymbol{u} \cdot \nabla \omega=\frac{1}{R e} \nabla^{2} \omega .
$$


The vorticity flux through a curvilinear arc $A E$ is defined as

$$
F=\int_{A}^{E} \boldsymbol{R} \cdot \boldsymbol{n} \mathrm{d} s,
$$

where $\boldsymbol{n}$ is a unit vector directed along the left normal to the integration arc. Golubkin $\&$ Sizykh (1987) showed that $\boldsymbol{R}$ is directed along lines $\boldsymbol{u}^{2} / 2+p=$ const. It can be easily shown that the vorticity flux is equal to the difference between the total pressures at the arc ends (Chernyshenko 1998):

$$
\int_{A}^{E} \boldsymbol{R} \cdot \boldsymbol{n} \mathrm{d} s=H(E)-H(A), \quad H=\boldsymbol{u}^{2} / 2+p .
$$

Indeed,

Taking into account that

$$
\frac{\partial H}{\partial x}=u \frac{\partial u}{\partial x}+v \frac{\partial v}{\partial x}+\frac{\partial p}{\partial x} .
$$

$$
\frac{\partial v}{\partial x}=\omega+\frac{\partial u}{\partial y}
$$

and using the $x$-component of the momentum equation from (2.1) we obtain

$$
\frac{\partial H}{\partial x}=v \omega-\frac{1}{R e} \frac{\partial \omega}{\partial y}=R_{y} .
$$

Here, the identity

$$
\nabla^{2} u=-\frac{\partial \omega}{\partial y}
$$

following from the continuity equation was used. Similarly

$$
\frac{\partial H}{\partial y}=-u \omega+\frac{1}{R e} \frac{\partial \omega}{\partial x}=-R_{x} .
$$

Substituting these expressions for $\left(R_{x}, R_{y}\right)=\boldsymbol{R}$ in (2.3) immediately proves that formula (2.4) is valid.

From this formula it follows that the vorticity flux though any closed contour equals zero. This condition is invariant in the sense that it is valid for arbitrary Reynolds number. Therefore, it must also hold for asymptotic solutions as $R e \rightarrow \infty$. This provides additional information on the asymptotic solutions, which may be used to determine them uniquely as Batchelor (1956) did when the well-known PrandtlBatchelor theorem was proved.

Provided that $u_{\infty}=$ const $\neq 0$ it is possible to derive another invariant condition valid for arbitrary Reynolds number, namely that on any contour enclosing the body the vorticity necessarily changes its sign, that is it cannot be only positive or only negative on the contour. This can be explained in the following way. Let us assume that on a certain contour enclosing the body the vorticity is positive. Since $u_{\infty}=$ const, the vorticity at infinity is zero. Since vorticity is governed by the Helmholtz equation, from the maximum principle (Courant 1962) it follows that the vorticity is positive everywhere outside the contour. Hence, it is also positive on a straight line perpendicular to $\boldsymbol{u}_{\infty}$, which we choose to be so far downstream that on this line $\boldsymbol{u}$ is quite close to $\boldsymbol{u}_{\infty}$ and, hence, is everywhere almost perpendicular to the line. It is now fairly obvious that the vorticity flux through this line is not zero. This can be demonstrated using the linearized Helmholtz equation with $\boldsymbol{u} \approx \boldsymbol{u}_{\infty}=$ const which can 


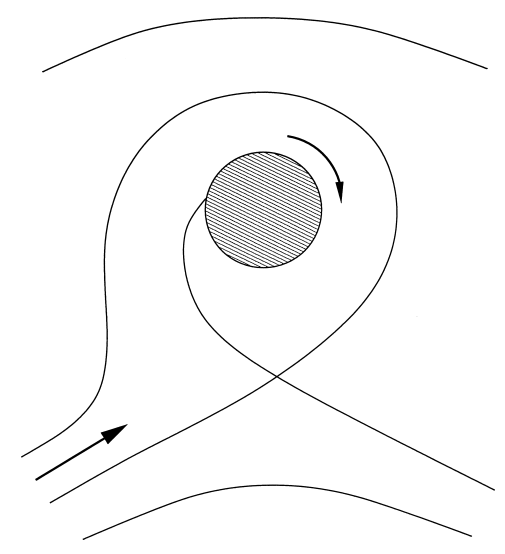

Figure 1. General flow topology.

be solved in quadratures. The rigorous mathematical proof for non-constant $\boldsymbol{u}$ was given by S. B. Kuksin (1997, personal communication). By the theorem formulated above this non-zero flux through the line is equal to the difference between the total pressure at infinity at each end of the line, which contradicts the condition $\boldsymbol{u}_{\infty}=$ const.

Note that this result (that vorticity necessarily changes sign on the contour enclosing the body) cannot be proved for contours not enclosing the body since in that case the maximum principle cannot be used, it cannot be proved for the case of a shear flow at infinity since in that case the difference between total pressures is infinite and there is no contradiction, and it cannot also be proved when $u_{\infty}=0$ since in that case we cannot choose a line on which the velocity vector is everywhere directed from one side of the line to the other.

The condition of vorticity necessarily changing sign on a contour enclosing the body and the condition of zero vorticity flux through a closed contour will be used in the subsequent sections.

\section{Strong suction}

Let the suction velocity $V_{w}$ be of order unity as $R e \rightarrow \infty$. Further let $V_{w}$ be large enough so that there are no closed streamlines. Then outside the boundary layer the flow will be potential, with no closed streamlines. This flow depends on one parameter, namely circulation $\Gamma$. The flow pattern is shown in figure 1 .

It is easy to verify that the characteristic thickness of the boundary layer in that case is $1 / R e$ and the governing equations in the boundary layer are

$$
V \frac{\partial U}{\partial Y}=\frac{\partial^{2} U}{\partial Y^{2}}, \quad \frac{\partial V}{\partial Y}=0,
$$

where $U$ and $V$ are the tangential and normal velocity components respectively, and $R e^{-1} Y$ is the coordinate normal to the wall. For a given distribution of suction velocity, $V=V_{w}(X)<$ const $<0$ at $Y=0$, from (3.1b) it follows that $V=V_{w}(X)$ everywhere inside the boundary layer.

To solve $(3.1 a)$ we, first, need to specify the wall velocity $U=U_{w}(X)$ at $Y=0$. The second boundary condition follows from matching with the inviscid flow region outside the boundary layer:

$$
U=U_{e}(X) \quad \text { at } \quad Y=\infty .
$$


Generally, $U_{e}(X)$ depends on the distribution of the suction velocity $V_{w}(X)$ and the circulation $\Gamma$. The solution satisfying these conditions is

$$
U=U_{e}(X)+\left(U_{w}(X)-U_{e}(X)\right) \exp \left(V_{w}(X) Y\right) .
$$

The vorticity in the boundary layer is

$$
\omega=-\operatorname{Re} \frac{\partial U}{\partial Y}=\operatorname{Re}\left(U_{e}(X)-U_{w}(X)\right) V_{w}(X) \exp \left(V_{w}(X) Y\right) .
$$

In particular, on the wall

$$
\omega_{w}=\operatorname{Re}\left(U_{e}(X)-U_{w}(X)\right) V_{w}(X) .
$$

Higher-order terms can be constructed quite easily. The solution obtained is not unique as it depends on the arbitrary parameter $\Gamma$ which is not known a priori. It is interesting to note that the method of matched asymptotic expansions, in its straightforward form used here to construct the solution, fails to detect any inconsistency whatever value of circulation $\Gamma$ is taken. Meanwhile it is easily seen that at least solutions with very large $\Gamma$, for which $\left|U_{e}(X)\right|>\left|U_{w}(X)\right|$, are not correct because for these solutions the vorticity on the wall does not change sign.

For certain distributions of wall velocity the invariant condition of vorticity being not strictly positive or strictly negative on a closed contour determines the circulation uniquely. Let $U_{w}(X)=U_{e 0}(X)+W, W=$ const, where $U_{e 0}(X)$ is equal to $U_{e}(X)$ for $\Gamma=0$. For the case of $V_{w}=$ const an explicit expression for $U_{e}(X)$ is given in the next section. Since $U_{e}(X)=U_{e 0}(X)+\Gamma / 2 \pi r_{0}$, (3.2) takes the form $\omega_{w}=$ $\operatorname{Re}\left(\Gamma / 2 \pi r_{0}-W\right) V_{w}(X)$. Therefore, the invariant condition is satisfied only provided that $\Gamma=2 \pi r_{0} W$.

Although in the general case of arbitrary $U_{w}(X)$ the invariant condition does not give $\Gamma$, the result obtained indicates the possibility that the true asymptotic solution is always unique, but that the usual asymptotic technique is not sufficient for determining this solution. For suction of order less than unity this is demonstrated in the following sections. It is also shown that this unusual situation is associated with exponentially small terms in the asymptotic expansions.

\section{Boundary layer with suction of order $1 / \sqrt{R e}$}

Consider a rotating circular cylinder of unit radius in a uniform flow with the free-stream velocity $u_{\infty}$. Let $U_{w}$ be the circumferential velocity of the cylinder surface and $V_{w}$ the suction velocity assumed to be constant over the cylinder surface. Let $V_{w}$ be a quantity of order $1 / \sqrt{R e}$, that is $V_{w}=-a / \sqrt{R e}$, where $a=O(1)$ and $a>0$. Introduce a curvilinear coordinate system with $X$ measured along the cylinder surface and scaled coordinate $Y=\operatorname{Re}^{1 / 2}(r-1)$ in the normal direction, as shown in figure 2 . The equation for the stream function $\Psi$ in the boundary layer takes the form

$$
\frac{\partial \Psi}{\partial Y} \frac{\partial^{2} \Psi}{\partial X \partial Y}-\frac{\partial \Psi}{\partial X} \frac{\partial^{2} \Psi}{\partial Y^{2}}=\frac{\partial^{3} \Psi}{\partial Y^{3}}+U_{e} \frac{\mathrm{d} U_{e}}{\mathrm{~d} X},
$$

where $U_{e}$ is the velocity at the outer edge of the boundary layer.

The flow outside the boundary layer is inviscid to the leading order. Since the suction is small, it does not influence the leading-order inviscid solution which is known to depend on the circulation $\Gamma$. When $\Gamma$ is large enough then a region with closed streamlines is formed around the cylinder. The vorticity along streamlines is constant and must be zero, since otherwise there would be a closed contour on which 


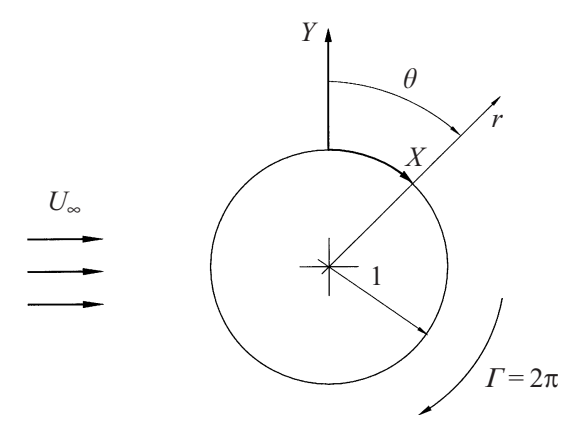

FIGURE 2. Coordinate system for a rotating cylinder.

the vorticity does not change sign. Therefore, the flow outside the boundary layer is potential.

It is convenient to prescribe the circulation in the outer flow and consider the wall velocity as an unknown parameter. Let us set $\Gamma=2 \pi$ which effectively means that the velocity components are made non-dimensional by reference to $\widehat{\Gamma} / r_{0}$ where $\widehat{\Gamma}$ is dimensional circulation and $r_{0}$ is cylinder radius. All the coordinates are made non-dimensional with $r_{0}$.

Then, in the inviscid flow outside the boundary layer the stream function $\psi$ takes the form

$$
\psi=u_{\infty}\left(r-\frac{1}{r}\right) \cos \theta+\log r,
$$

hence

$$
U_{e}=\left.\frac{\partial \psi}{\partial r}\right|_{r=1}=1+2 u_{\infty} \cos X
$$

and

$$
U_{e} \frac{\mathrm{d} U_{e}}{\mathrm{~d} X}=-2 u_{\infty} \sin X-2 u_{\infty}^{2} \sin 2 X .
$$

Thus, the problem for the boundary layer takes the form

$$
\frac{\partial \Psi}{\partial Y} \frac{\partial^{2} \Psi}{\partial X \partial Y}-\frac{\partial \Psi}{\partial X} \frac{\partial^{2} \Psi}{\partial Y^{2}}=\frac{\partial^{3} \Psi}{\partial Y^{3}}-2 u_{\infty} \sin X-2 u_{\infty}^{2} \sin 2 X
$$

with the boundary conditions being

$$
\left.\frac{\partial \Psi}{\partial Y}\right|_{Y=0}=U_{w},\left.\quad \frac{\partial \Psi}{\partial X}\right|_{Y=0}=a,\left.\quad \frac{\partial \Psi}{\partial Y}\right|_{Y \rightarrow \infty}=U_{e} .
$$

In addition, the periodicity condition for the velocity components

$$
\left.\frac{\partial \Psi}{\partial X}\right|_{X+2 \pi}=\left.\frac{\partial \Psi}{\partial X}\right|_{X},\left.\quad \frac{\partial \Psi}{\partial Y}\right|_{X+2 \pi}=\left.\frac{\partial \Psi}{\partial Y}\right|_{X}
$$

must hold.

To the authors' knowledge, there are no rigorous mathematical results concerning the uniqueness and existence of the solution of this problem. However, on the basis of some relevant results mentioned earlier, it can be expected that for $a=0$ this problem has no solution for arbitrary $U_{w}$ and $U_{e}$ (hence, for arbitrary $\Gamma$ ). Then, the solvability condition can be used for determining $U_{w}$ for a given $\Gamma$ or vice versa. For $a>0$ this problem has a solution for any $\Gamma$ and $U_{w}$, at least within a certain $\Gamma$ range when there is no separation. Hence, without the use of some invariant condition or more 
subtle analysis, the straightforward asymptotic solution is not unique. We illustrate this in the rest of this section for the case of small free-stream velocity.

Following Glauert (1957) let us assume that the free-stream velocity $u_{\infty}$ is small and represent the solution of this problem as

$$
\Psi=\Psi_{0}+u_{\infty} \Psi_{1}+u_{\infty}^{2} \Psi_{2}+\cdots .
$$

In this formulation the circumferential velocity of the cylinder surface $U_{w}$ should be found as a part of the solution. We will seek it in the form

$$
U_{w}=U_{w 0}+u_{\infty} U_{w 1}+u_{\infty}^{2} U_{w 2}+\cdots .
$$

In the case of zero suction considered by Glauert (1957) the solvability condition for the periodic boundary layer derived by Batchelor (1956) and Squire (1956) gives $U_{w 0}=1$, but in our case this condition does not apply.

For the leading-order term $\Psi_{0}$ in the expansion of the stream function the following boundary-value problem holds:

$$
\begin{gathered}
\frac{\partial \Psi_{0}}{\partial Y} \frac{\partial^{2} \Psi_{0}}{\partial X \partial Y}-\frac{\partial \Psi_{0}}{\partial X} \frac{\partial^{2} \Psi_{0}}{\partial Y^{2}}=\frac{\partial^{3} \Psi_{0}}{\partial Y^{3}} \\
\left.\frac{\partial \Psi_{0}}{\partial Y}\right|_{Y=0}=U_{w 0} ;\left.\quad \frac{\partial \Psi_{0}}{\partial X}\right|_{Y=0}=a ;\left.\quad \frac{\partial \Psi_{0}}{\partial Y}\right|_{Y \rightarrow \infty}=1 .
\end{gathered}
$$

Its solution satisfying the periodicity condition for the velocity components has the form

$$
\Psi_{0}=Y+a X+\left(1-U_{w 0}\right) \mathrm{e}^{-a Y} / a .
$$

This solution exists for any value of $U_{w 0}$ and, therefore, $U_{w 0}$ cannot be determined from a solvability condition. However, since in this solution the vorticity at the wall is $a\left(1-U_{w 0}\right)$ then from the invariant condition of the vorticity on a contour not being of a fixed sign it follows that $U_{w 0}=1$.

The boundary-value problem for the next-order term $\Psi_{1}$ proves to be

$$
\begin{gathered}
\frac{\partial^{2} \Psi_{1}}{\partial X \partial Y}-a \frac{\partial^{2} \Psi_{1}}{\partial Y^{2}}=\frac{\partial^{3} \Psi_{1}}{\partial Y^{3}}+\mathrm{i}\left(\mathrm{e}^{\mathrm{i} X}-\mathrm{e}^{-\mathrm{i} X}\right), \\
\left.\frac{\partial \Psi_{1}}{\partial Y}\right|_{Y=0}=U_{w 1},\left.\quad \frac{\partial \Psi_{1}}{\partial X}\right|_{Y=0}=0,\left.\quad \frac{\partial \Psi_{1}}{\partial Y}\right|_{Y \rightarrow \infty}=\mathrm{e}^{\mathrm{i} X}+\mathrm{e}^{-\mathrm{i} X} .
\end{gathered}
$$

A periodic solution to this problem may be sought in the form

$$
\Psi_{1}=f_{0}(Y)+f_{1}(Y) \mathrm{e}^{\mathrm{i} X}+\overline{f_{1}(Y)} \mathrm{e}^{-\mathrm{i} X},
$$

which gives after a substitution

$$
\begin{gathered}
f_{0}^{\prime \prime \prime}+a f_{0}^{\prime \prime}=0, \quad f_{0}^{\prime}(0)=U_{w 1}, \quad f_{0}(0)=0, \quad f_{0}^{\prime}(\infty)=0, \\
f_{1}^{\prime \prime \prime}+a f_{1}^{\prime \prime}-\mathrm{i}\left(f_{1}^{\prime}-1\right)=0, \quad f_{1}^{\prime}(0)=0, \quad f_{1}(0)=0, \quad f_{1}^{\prime}(\infty)=1,
\end{gathered}
$$

where the overbar denotes complex conjugate quantities. It is easily deduced that

$$
f_{0}=\frac{U_{w 1}}{a}\left(1-\mathrm{e}^{-a Y}\right), \quad f_{1}=-\frac{1}{\lambda_{1}} \mathrm{e}^{\lambda_{1} Y}+Y+\frac{1}{\lambda_{1}},
$$

where $\lambda_{1}=\left(-a-\sqrt{a^{2}+4 i}\right) / 2$ and the real part of $\lambda_{1}$ is negative. It is easily seen that the $n$th term in the expansion of the stream function will contain an additive term 
of the form $f_{n}(Y) \mathrm{e}^{\mathrm{i} n}$ and its complex conjugate $\overline{f_{n}(Y)} \mathrm{e}^{-\mathrm{i} n X}$. Each function $f_{n}(Y)$ satisfies the equation

$$
f_{n}^{\prime \prime \prime}+a f_{n}^{\prime \prime}-\mathrm{in} f_{n}^{\prime}=F_{n}(Y)
$$

where the right-hand side is determined from the previous approximations. The solution of this equation includes a term of the form $C_{n} \mathrm{e}^{\mathrm{i} n X} \mathrm{e}^{\lambda_{n} Y}$ where $\lambda_{n}$ is the solution of the quadratic equation

$$
\lambda_{n}^{2}+a \lambda_{n}-\mathrm{i} n=0
$$

and we have to choose the root with negative real part.

As it can be seen from (4.1), the solution exists for any $U_{w 1}$. Contrary to that Glauert's (1957) solution with no suction determines $U_{w 1}$ and all subsequent terms in the expansion of $U_{w}$ uniquely. The reason is that for $a=0$ we can satisfy both $f_{0}^{\prime}(0)=U_{w 1}$ and $f_{0}^{\prime}(\infty)=0$ boundary conditions simultaneously only when $U_{w 1}=0$ which leads to uniqueness of Glauert's solution.

Glauert obtained one more term in $u_{\infty}$ :

$$
U_{w}=1+3 u_{\infty}^{2}+\cdots
$$

but we will limit our analysis to linear terms only.

Taking the second derivative of the solution for $\Psi$ with respect to $Y$ gives the vorticity distribution in the boundary layer

$$
\omega=R e^{1 / 2} \frac{\partial^{2} \Psi}{\partial Y^{2}}=-\operatorname{Re}^{1 / 2} u_{\infty}\left(a U_{w 1} \mathrm{e}^{-a Y}+\lambda_{1} \mathrm{e}^{\mathrm{i} X} \mathrm{e}^{\lambda_{2} Y}+\bar{\lambda}_{1} \mathrm{e}^{-\mathrm{i} X} \mathrm{e}^{\bar{\lambda}_{1} Y}\right)+\cdots
$$

It tends to zero exponentially at the outer edge of the boundary layer. As $Y \rightarrow \infty$ in (4.3) the main term of the vorticity is $-u_{\infty} R e^{1 / 2} a U_{w 1} \mathrm{e}^{-a Y}$ and it does not change sign on a contour enclosing a body apart from the case when $U_{w 1}=0$. Hence, here again the use of an invariant condition determines a unique solution, while the usual technique gives a non-unique solution.

However, the invariant condition throws no light on the reasons underlying this behaviour of the asymptotic expansion, and we still need a more profound asymptotic analysis to be performed. For this reason we will return to the case of suction of order $R e^{-1 / 2}$ after analysing the case of even weaker suction, which can be considered without the use of invariant conditions.

If we consider a 'weak suction' with $V_{w} \ll R e^{-1 / 2}$, then a new region which is wider than the conventional boundary layer should be studied. Indeed, in this case $a=-R e^{1 / 2} V_{w}$ is small and (4.3) suggests the existence of a layer where $r-1=R e^{-1 / 2} Y$ is of order $R e^{-1 / 2} / a$. A suitable normal variable in this layer is

$$
\widetilde{Y}=a Y=a e^{1 / 2}(r-1) .
$$

When the suction velocity is diminished to $V_{w}=O\left(R e^{-1}\right)$ the thickness of the new layer becomes comparable with the cylinder radius. This flow regime will be considered in the following section.

\section{The flow with a suction of order $1 / R e$}

The flow outside the boundary layer is inviscid and in the main approximation may be described by the equation

$$
\Delta \psi=-\omega(\psi),
$$

where it is taken into account that the vorticity $\omega$ is constant along each streamline. 


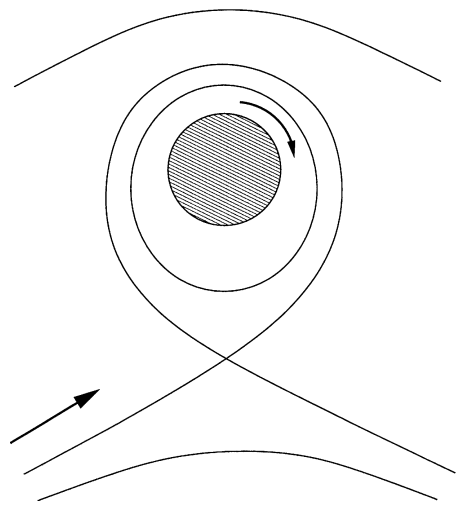

FIGURE 3. Flow topology without suction.

To solve this problem it is necessary to determine the vorticity distribution $\omega=$ $\omega(\psi)$. On the streamlines coming from infinity, this distribution is determined by the boundary condition at infinity, and usually $\omega(\psi)=0$. In regions of closed streamlines $\omega(\psi)=$ const according to the Prandtl-Batchelor theorem (Batchelor 1956). This theorem cannot be directly used for the flow past a rotating cylinder with a weak suction because in this case the streamlines are not exactly closed, but have a helical shape.

Nevertheless, taking into account that suction is weak, one can expand the solution in terms of $1 / R e$, and the leading-order approximation will represent a flow with closed streamlines. The flow pattern for the main term is shown in figure 3. Here again, the use of the condition that vorticity should change its sign on a closed contour gives immediately that on these closed streamlines $\omega(\psi)=0$. However, in this case an explicit asymptotic analysis, which is performed in the rest of this section, gives substantial additional information.

As was shown above, the vorticity flux through any closed contour $C$ must be zero:

$$
\int_{C}\left(\boldsymbol{u} \omega-R e^{-1} \nabla \omega\right) \boldsymbol{n} \mathrm{d} s=0 .
$$

Choose a closed streamline of the leading-order approximation as the integration contour $C$. If this streamline is situated in the inviscid region where $\omega=\omega(\psi)$ then (5.1) becomes

$$
\omega \int_{C}(\boldsymbol{u}, \boldsymbol{n}) \mathrm{d} s-R e^{-1} \frac{\mathrm{d} \omega}{\mathrm{d} \psi} \int_{C}(\boldsymbol{u}, \tau) \mathrm{d} s=0,
$$

where $\tau$ is the unit vector tangent to $C$. It gives the following equation for the vorticity distribution in the region under consideration:

$$
q \omega-\operatorname{Re}^{-1} \Gamma(\psi) \frac{\mathrm{d} \omega}{\mathrm{d} \psi}=0 .
$$

Here, $q$ is the fluid flux through the cylinder surface. When the suction velocity is of order $1 / R e$, we can write $q=R e^{-1} Q$ with $Q=O(1)$ and the equation for $\omega$ becomes

$$
Q \omega-\Gamma(\psi) \frac{\mathrm{d} \omega}{\mathrm{d} \psi}=0,
$$

with circulation $\Gamma(\Psi)$ being related to vorticity $\omega$ via the Stokes theorem.

Equation (5.2) will be used in the region with closed streamlines. For the rest 
of the inviscid flow $\omega=0$ due to the boundary conditions at infinity. Denote the stream function on the dividing streamline by $\psi^{*}$. The vorticity might experience a jump across the dividing streamline leading to the formation of a thin mixing layer. Vorticity $\omega$ in this layer is governed by the equation

$$
\frac{\partial \omega}{\partial s}=u(s) \frac{\partial^{2} \omega}{\partial \Psi^{2}}
$$

which is written in the Mises variables where $\Psi=R e^{1 / 2}\left(\psi-\psi^{*}\right), s$ is the distance measured along the dividing streamline from the stagnation point, and $u(s)$ is the fluid velocity on the dividing streamline.

The boundary conditions for (5.3) are

$$
\begin{array}{ll}
\omega \rightarrow 0 & \text { as } \quad \Psi \rightarrow+\infty, \\
\omega \rightarrow \Omega & \text { as } \quad \Psi \rightarrow-\infty .
\end{array}
$$

Here $\Omega$ is the limiting value of the vorticity when the dividing streamlines is approached from the closed streamline region:

$$
\Omega=\lim _{\psi \rightarrow \psi^{*}-0} \omega(\psi) .
$$

To complete the formulation of the problem we note that the mixing layer passes near the stagnation point. In a small vicinity of this point the flow is locally inviscid (see for example Neiland \& Sychev 1966), and the vorticity is constant along all streamlines. Taking into account that part of them returns back into the mixing layer, the following condition should hold:

$$
\omega(0, \Psi)=\omega(\tilde{S}, \Psi) H(-\Psi),
$$

where $\tilde{S}$ is the total length of the streamline $\psi=\psi^{*}$ and $H$ is the step function

$$
H(z)= \begin{cases}1 & \text { if } z \geqslant 0 \\ 0 & \text { if } z<0 .\end{cases}
$$

Let us introduce new variables $t$ and $\eta$ :

$$
t=\frac{1}{T} \int_{0}^{s} u(z) \mathrm{d} z, \quad \eta=\frac{\psi}{\sqrt{T}}
$$

where

$$
T=\int_{0}^{\tilde{S}} u(z) \mathrm{d} z
$$

In these variables equation (5.3) becomes the heat equation and the problem for $\omega$ takes the form

$$
\left.\begin{array}{l}
\frac{\partial \omega}{\partial t}=\frac{\partial^{2} \omega}{\partial \eta^{2}}, \\
\omega(0, x)=\omega(1, x) H(-x), \\
\omega \rightarrow 0 \text { as } \eta \rightarrow+\infty, \\
\omega \rightarrow \Omega \text { as } \eta \rightarrow-\infty .
\end{array}\right\}
$$

From the results obtained by Chernyshenko (1995) it follows that (5.4) has a solution 
only if $\Omega=0$. This gives the following boundary condition for the equation (5.2):

$$
\omega\left(\psi^{*}\right)=0 .
$$

Equation (5.2) may be solved quite easily if we assume the vorticity to be small. Then in the first approximation $\Gamma=\mathrm{const}=2 \pi$. In addition, let the normal velocity component on the cylinder surface be $V_{w}=-R e^{-1} \alpha, \quad \alpha \sim 1, \quad \alpha>0$. Then $Q=$ $-2 \pi \alpha$. Under this assumption the equation takes a simple form:

$$
\alpha \omega+\frac{\mathrm{d} \omega}{\mathrm{d} \psi}=0
$$

which gives

$$
\omega=C \mathrm{e}^{-\alpha \psi} .
$$

Note that under this assumption of small vorticity the value of $\psi^{*}$ can be found from the potential solution for a cylinder in the uniform flow $u_{\infty}$ with circulation $\Gamma=2 \pi$, and it is

where

$$
\psi^{*}=u_{\infty}\left(\frac{1}{r^{*}}-r^{*}\right)+\log r^{*}
$$

$$
r^{*}=\frac{1+\sqrt{1-4 u_{\infty}^{2}}}{2 u_{\infty}} .
$$

From the boundary condition (5.5) the factor $C$ in (5.6) is zero as is the vorticity throughout the entire flow field outside the boundary layer. (This is, of course, the same result as obtained above using the invariant condition.) Thus, the circulation is not changed in this region and its magnitude at infinity is equal to the circulation on the outer edge of the boundary layer. It is easy to verify that suction of order $R e^{-1}$ does not manifest itself in the viscous boundary layer near the cylinder surface. Therefore, the usual analysis applies, and the solvability condition yields a unique circulation given by the same formula as in the case of no suction.

Let us consider now the case when the vorticity far upstream is $\Omega_{\infty} \neq 0$. No invariant condition can be used in this case. For simplicity we assume $\Omega_{\infty} \ll 1$. In that case the main term for the flow on the body scale is the same, but the boundary condition for the mixing layer is $\omega \rightarrow \Omega_{\infty}$ as $x \rightarrow+\infty$. Accordingly, the solution in the mixing layer exists only if $\Omega=\Omega_{\infty}$. Therefore, inside the region of closed streamlines instead of $\omega=0$ we have

$$
\omega=\Omega_{\infty} \mathrm{e}^{-\alpha\left(\psi-\psi^{*}\right)} .
$$

The difference between the circulation along the closed contour immediately adjacent to the mixing layer and that at the outer edge of the boundary layer near the cylinder surface equals, by the Stokes theorem, the integral of $\omega$ over the closed streamline region. For a given circulation outside the region of closed streamlines this will lead to different values of $U_{w}$. Let us suppose now that $\alpha$ is large, and consider the variation in $\Omega_{\infty}$ which ensures a variation of order unity in $U_{w}$. From (5.7) it follows that this variation is exponentially small with respect to $\alpha$. More exactly,

$$
\Delta \Omega_{\infty} \sim \alpha \exp \left(-\alpha \psi^{*}\right) \Delta U_{w}=-\operatorname{Re} V_{w} \exp \left(\operatorname{Re} V_{w} \psi^{*}\right) \Delta U_{w} .
$$

Note that $V_{w}<0$. This implies that for suction stronger than $O(1 / R e)$ asymptotic solutions with different $U_{w}$ for the same $\Gamma$ will correspond to different, but exponentially small with respect to $R e$, values of $\Omega_{\infty}$. Here $\Gamma$ on the body scale only is implied since on larger scales the circulation depends on the contour. The high sensitivity 


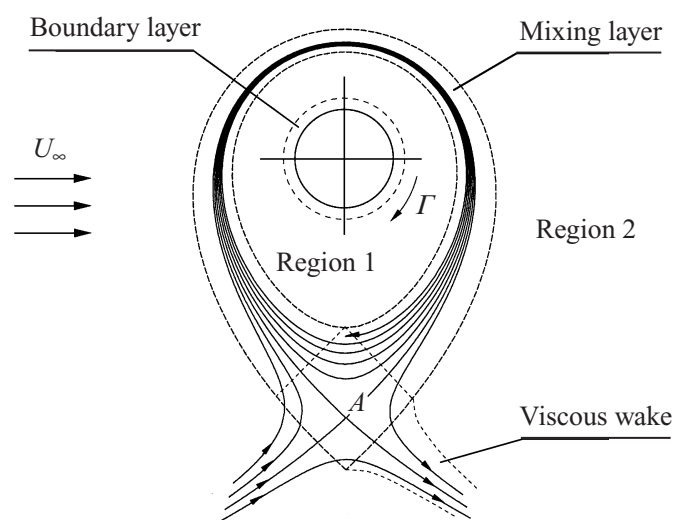

Figure 4. The structure of the flow past a rapidly rotating cylinder with weak suction.

of the solution to the upstream level of vorticity has a simple physical explanation. Consider again figure 1. Two streamlines going from upstream to the saddle point form a pocket in which the vorticity advected by the flow is trapped. Inside the pocket vorticity is advected everywhere toward the cylinder. However, according to the theorem from $\S 2$, the total vorticity flux to the body should be zero. Therefore, this advection has to be balanced by vorticity diffusion. The advection rate is proportional to the vorticity itself while the diffusion rate is proportional to the vorticity gradient, divided by $R e$, as expressed by (5.1). This leads to the exponential behaviour of the vorticity and, hence, to high sensitivity of the solution to the upstream vorticity level.

A standard asymptotic technique cannot distinguish exponentially small quantities, and therefore these asymptotic solutions will look like non-unique solutions for the case of a uniform flow at infinity. This implies also that to determine the unique solution corresponding to $\Omega_{\infty}=0$ exponentially small terms should be explicitly taken into account. An attempt to do this is made in the following section.

\section{Vorticity behaviour outside the boundary layer when suction is of order $1 / \sqrt{R e}$}

Let us now consider the flow with suction velocity $v_{w} \sim 1 / \sqrt{R e}$, which corresponds to $a=-V_{w} \sqrt{R e} \sim 1$. The analysis in this section uses the fact that everywhere outside the boundary layer the vorticity is exponentially small and does not affect the velocity field in algebraic approximations with respect to Re. Helmholtz's equation (2.2) in this case becomes linear with the velocity field $\boldsymbol{u}$ given by the well-known potential solution (slightly perturbed by the displacement effect of the boundary layer). The circulation in the potential flow remains constant, and using suitable scaling it may be chosen to be $2 \pi$. The streamline pattern of such a flow with a weak suction is shown in figure 4.

For analysing the vorticity behaviour in region 1 it is convenient to use a new coordinate system $(\phi, \psi)$, where $\phi$ and $\psi$ are the velocity potential and stream function. In this coordinate system equation (2.2) may be written as

$$
\frac{\partial \omega}{\partial \phi}=\epsilon^{2}\left(\frac{\partial^{2} \omega}{\partial \phi^{2}}+\frac{\partial^{2} \omega}{\partial \psi^{2}}\right)
$$


where $\epsilon=1 / \sqrt{R e}$. We will seek the solution of equation (6.1) in the form

$$
\omega=\Omega_{0}+\sum_{n=0}^{\infty} C_{n} \mathrm{e}^{\alpha_{n} \phi} \mathrm{e}^{\lambda_{n} \psi / \epsilon}, \quad \Omega_{0}=\text { const. }
$$

If substituted into (6.1) this gives

$$
\epsilon^{2} \alpha_{n}^{2}-\alpha_{n}+\lambda_{n}^{2}=0
$$

and we have

Therefore

$$
\alpha_{n}=\lambda_{n}^{2}+\epsilon^{2} \lambda_{n}^{4}+\cdots
$$

$$
\omega=\Omega_{0}+\sum_{n} C_{n} \mathrm{e}^{\lambda_{n} \psi / \epsilon}\left(\mathrm{e}^{\lambda_{n}^{2} \phi}+O\left(\epsilon^{2}\right)\right) .
$$

Note the form of asymptotic expansion (6.2). Since equation (6.1) is linear, the order of coefficients $C_{n}$ does not matter and the order of magnitude of each term at each point depends on the magnitude of the exponent $\mathrm{e}^{\lambda_{n} \psi / \epsilon}$ at that point. By varying the coefficients we may make some of the terms to be of the same order of magnitude in the vicinity of a certain curve $\psi=$ const, but in any case these terms have different orders of magnitude at all other points in space. In addition, all exponents may be multiplied by some standard asymptotic expansion. We will handle only the first terms of these expansions, which are important for our further consideration.

In region 1 (figure 4 ) the following periodicity condition must hold:

$$
\omega(\phi, \psi)=\omega(\phi+2 \pi, \psi+2 \pi a \epsilon)
$$

which implies that $\lambda_{n}$ in (6.2) should satisfy the following equation:

$$
\lambda_{n}^{2}+a \lambda_{n}=n \mathrm{i},
$$

where $n$ is an integer.

This equation is the same as equation (4.2) derived from the boundary layer analysis. Hence, by choosing properly coefficients $C_{n}$ in (6.2) we can match solutions for the boundary layer and region 1 . The matching also suggests that the roots of equation (6.3) should have a negative real part. Therefore

$$
\lambda_{n}=\frac{-a-\sqrt{a^{2}+4 n \mathrm{i}}}{2}
$$

and for the two leading coefficients in (6.2) we have

$$
C_{0}=-\frac{a u_{\infty} U_{w 1}}{\epsilon}+O\left(\frac{u_{\infty}^{2}}{\epsilon}\right), \quad C_{1}=-\frac{\lambda_{1} u_{\infty}}{\epsilon}+O\left(\frac{u_{\infty}^{2}}{\epsilon}\right) .
$$

It should be noted that $C_{-n}=\bar{C}_{n}$.

Solution (6.2) is valid in region 1. In region 2 (see figure 4) we have to take $\omega \equiv 0$ to satisfy boundary condition $\omega=0$ at infinity. To match solutions in regions 1 and 2 we need to introduce a mixing layer with thickness $1 / \sqrt{R e}$ along the dividing streamline $\psi^{*}$, which takes its origin at critical point $A$ (figure 4). The solution in the mixing layer may be represented in the form

$$
\omega=\sum_{n} C_{n} \mathrm{e}^{\lambda_{n} \psi^{*} / \epsilon} \omega_{n}
$$

where functions $\omega_{n}$ depend on $t=\phi$ and $\xi=\operatorname{Re}^{1 / 2}\left(\psi-\psi^{*}\right)$ as well as on the 
parameter $\epsilon=R e^{-1 / 2}$, and themselves may be expanded in powers of $\epsilon$. For the leading-order term of $\omega_{n}$ the following equation holds:

$$
\frac{\partial \omega_{n}}{\partial t}=\frac{\partial^{2} \omega_{n}}{\partial \xi^{2}}
$$

with the boundary conditions being

$$
\begin{array}{lll}
\omega_{n} \rightarrow \mathrm{e}^{\lambda_{n}^{2} t} \mathrm{e}^{\lambda_{n} \xi} & \text { as } & \xi \rightarrow-\infty, \\
\omega_{n}=o\left(\mathrm{e}^{\lambda_{n} \xi}\right) & \text { as } & \xi \rightarrow+\infty .
\end{array}
$$

To formulate the periodicity condition for the mixing layer one needs to consider the locally inviscid region which occupies a small vicinity around the critical point $A$; both dimensions of this region are of order $1 / \sqrt{R e}$ in $\phi$ and $\psi$ variables. Taking into account that the vorticity in this region remains constant along streamlines, and that a part of them 'comes' from the unperturbed free stream where $\omega=0$, we have

$$
\omega_{n}(0, \xi)=\omega_{n}(2 \pi, \xi+2 \pi a) H(-\xi) .
$$

Finally, as was shown above, vorticity flux through any contour enclosing the body must be zero. For a contour lying far enough outside the mixing layer this flux is determined by vorticity convection into the viscous wake. According to (6.7) all streamlines with $\xi<2 \pi a$ remain inside the mixing layer, while others go into the wake outside the critical point $A$, creating the vorticity flux. Since vorticity is constant along streamlines in the turning region, this flux equals the flux upstream of the region where streamlines turn, that is at $t=2 \pi$ with $2 \pi a<\xi<\infty$. Therefore, we have one more condition:

$$
\sum_{n} C_{n} \mathrm{e}^{\lambda_{n} \psi^{*} / \epsilon} \int_{2 \pi a}^{\infty} \omega_{n}(2 \pi, \xi) \mathrm{d} \xi=0 .
$$

To eliminate the shift $2 \pi a$ in the condition (6.7), instead of variable $\xi$ we introduce a new variable $\chi=\xi-a t$. As a result, we obtain the following problem for functions $\omega_{n}$ in the mixing layer:

$$
\begin{aligned}
& \frac{\partial \omega_{n}}{\partial t}=\frac{\partial^{2} \omega_{n}}{\partial \chi^{2}}+a \frac{\partial \omega_{n}}{\partial \chi}, \\
& \omega_{n}(0, \chi)=\omega_{n}(2 \pi, \chi) H(-\chi), \\
& \omega_{n} \sim \mathrm{e}^{\mathrm{i} n t} \mathrm{e}^{\lambda_{n} \chi}+\cdots \quad \text { as } \quad \chi \rightarrow-\infty, \\
& \omega_{n} \rightarrow 0 \quad \text { as } \chi \rightarrow+\infty \text {, }
\end{aligned}
$$

and the condition for zero vorticity flux now takes the form

$$
\sum_{n} C_{n} \mathrm{e}^{\lambda_{n} \psi^{*} / \epsilon} \int_{0}^{\infty} \omega_{n}(2 \pi, \chi) \mathrm{d} \chi=0
$$

We expect that the solution of problem (6.8)-(6.10) gives

$$
\omega_{n}-\mathrm{e}^{\mathrm{i} n t} \mathrm{e}^{\lambda_{n} \chi} \rightarrow A_{n} \quad \text { as } \quad \chi \rightarrow-\infty
$$

where $A_{n}$ are non-zero constants depending on the suction parameter $a$. Using the standard energy arguments it is easy to demonstrate that (6.8)-(6.9) has no solution with $\omega_{n} \rightarrow 0$ as $\chi \rightarrow+\infty$ and $\omega_{n} \rightarrow$ const $\neq 0$ as $\chi \rightarrow-\infty$. For this reason $\Omega_{0}$ is not independent from $C_{n}$. Instead,

$$
\Omega_{0}=\sum_{n} C_{n} \mathrm{e}^{\lambda_{n} \psi^{*} / \epsilon} A_{n}
$$




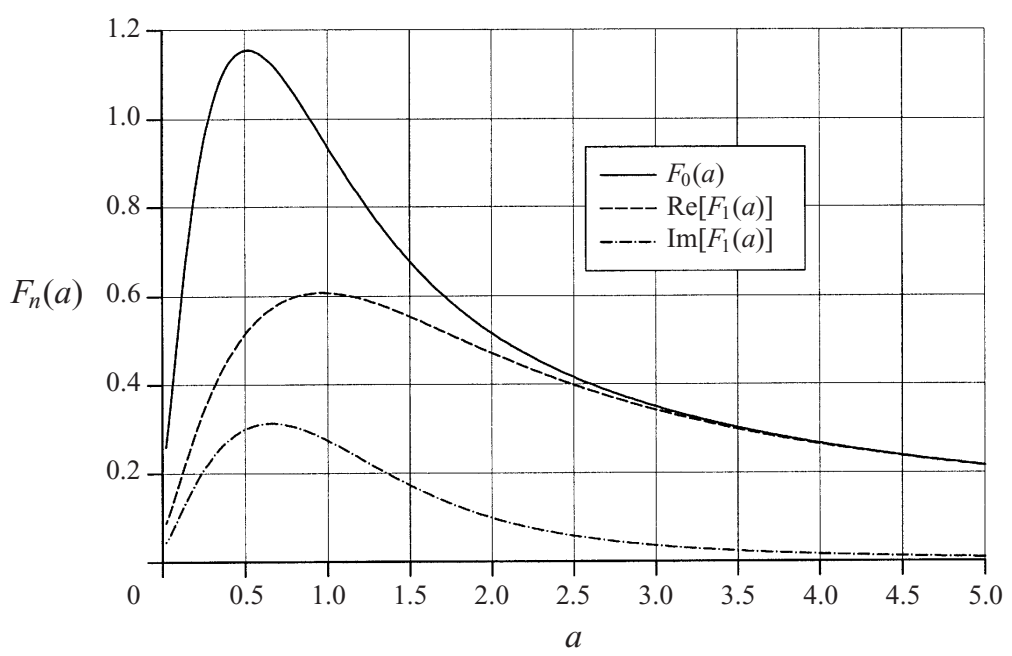

FIGURE 5. Behaviour of function $F_{n}(a)$.

Now, $\Omega_{0}$ is associated with a non-zero vorticity flux $2 \pi a \Omega_{0}$ toward the cylinder due to vorticity convection, and, of course, the same flux taken with an opposite sign should be convected into the wake since vorticity is conserved. It means that each function $\omega_{n}$ gives some non-zero contribution to the vorticity flux in the viscous wake. Indeed, let us consider equation (6.8) taking account of (6.12). After integration of this equation with respect to $t$ from 0 to $2 \pi$ and taking into account periodicity condition (6.9) we obtain

$$
\omega_{n}(2 \pi, \chi) H(\chi)=\frac{\partial^{2}}{\partial \chi^{2}} \int_{0}^{2 \pi} \omega_{n} \mathrm{~d} t+a \frac{\partial}{\partial \chi} \int_{0}^{2 \pi} \omega_{n} \mathrm{~d} t .
$$

Now integrate this expression with respect to $\chi$ from $-\infty$ to $\infty$. Using the second boundary condition (6.10) and (6.12) we have

$$
\int_{0}^{+\infty} \omega_{n}(2 \pi, \chi) \mathrm{d} \chi=-a A_{n} \int_{0}^{2 \pi} \mathrm{d} t .
$$

Thus, the flux of $\omega_{n}$ into the viscous wake is

$$
F_{n}=\int_{0}^{+\infty} \omega_{n}(2 \pi, \chi) \mathrm{d} \chi=-2 \pi a A_{n} .
$$

The dependence of $F_{n}=2 \pi a A_{n}$ on $a$, obtained from the numerical solution of (6.8)(6.10) for $n=0$ and $n=1$ is given in figure 5. As an example, in figure 6 and figure 7 functions $\mathrm{e}^{-\lambda_{n} \chi} \omega_{n}(2 \pi, \chi)$ are shown for $n=0$ and $n=1$ respectively.

Note that since the form of vorticity equation coincides with the form of the equation for temperature in the same flow (in the case of zero Eckert number), the results obtained here also give the solution for the temperature. In particular, the heat flux from the cylinder is $\sum_{n} C_{n} \mathrm{e}^{\lambda_{n} \psi^{*} / \epsilon} F_{n}$, although in this case we should take $\epsilon=1 / \sqrt{\operatorname{RePr}}$, where $\operatorname{Pr}$ is the Prandtl number, and $C_{n}$ should be found from the temperature distribution on the cylinder surface.

In fact, expansion (6.6) is asymptotic, because each exponent $\mathrm{e}^{\lambda_{n} \psi^{*} / \epsilon}$ is much less than the previous one. Coefficients $C_{n}, n \neq 0$, are of order unity with respect to the Reynolds number and defined by matching with the boundary layer on the cylinder 


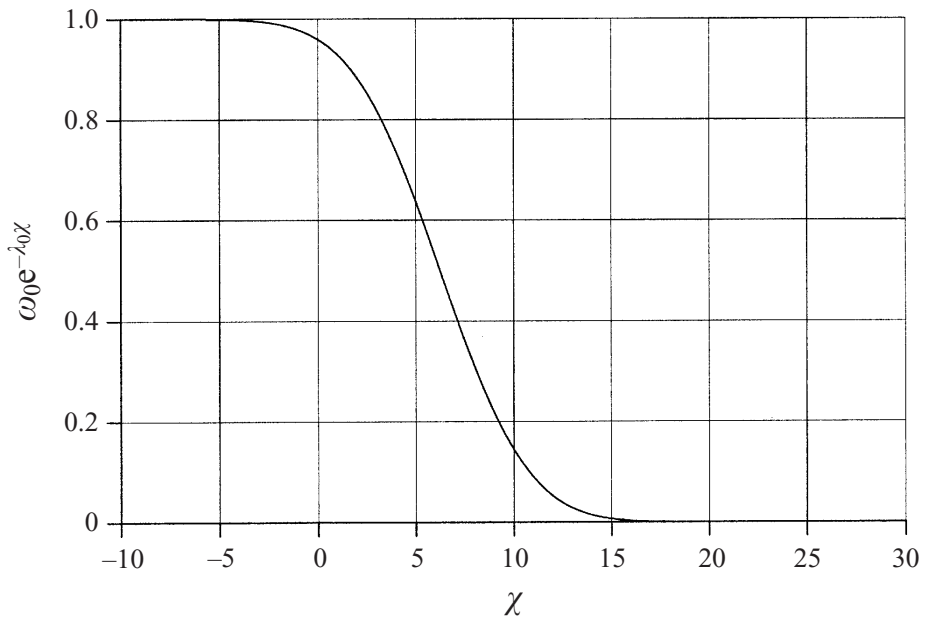

FIgURE 6. Profile of function $\omega_{0} \mathrm{e}^{-\lambda_{0} \chi}, t=2 \pi, a=1$.

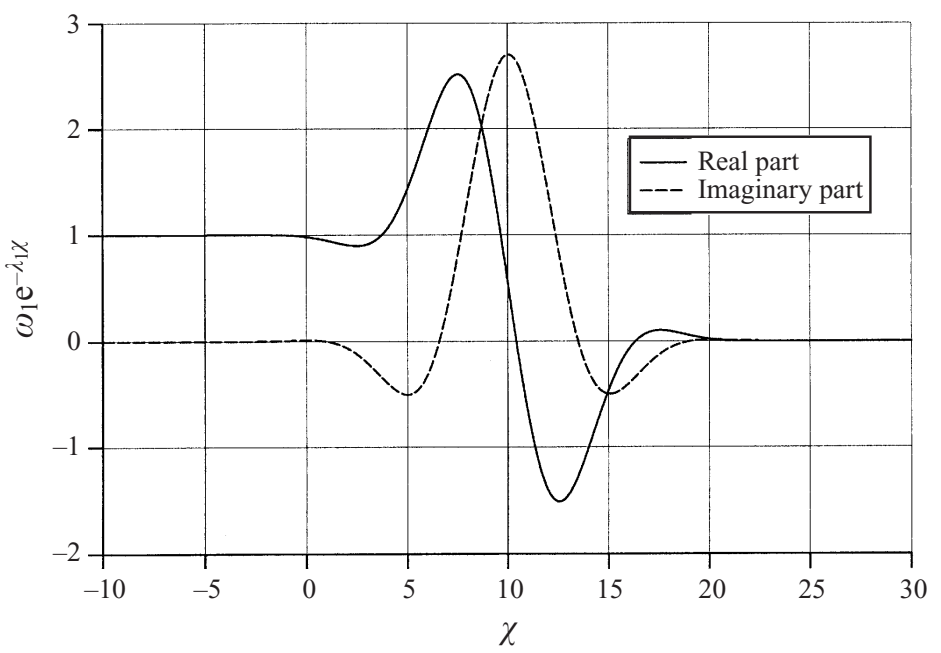

Figure 7. Profile of function $\omega_{1} \mathrm{e}^{-\lambda_{1} \chi}, t=2 \pi, a=1$.

surface. But we still have one free parameter, namely the coefficient $C_{0}$. Being proportional to $U_{w 1}$, it is not defined yet and the proper choice of this coefficient can ensure that (6.11) or, equivalently, $\Omega_{0}=0$ is satisfied. For the main approximation we have

$$
\begin{aligned}
C_{0} \mathrm{e}^{\lambda_{0} \psi^{*} / \epsilon} \int_{0}^{\infty} \omega_{0}(2 \pi, \chi) \mathrm{d} \chi+C_{1} \mathrm{e}^{\lambda_{1} \psi^{*} / \epsilon} \int_{0}^{\infty} \omega_{1}(2 \pi, \chi) \mathrm{d} \chi \\
=F_{0}(a) C_{0} \mathrm{e}^{\lambda_{0} \psi^{*} / \epsilon}+F_{1}(a) C_{1} \mathrm{e}^{\lambda_{1} \psi^{*} / \epsilon}=0,
\end{aligned}
$$

from which:

$$
C_{0}=-C_{1} \mathrm{e}^{\left(\lambda_{1}-\lambda_{0}\right) \psi^{*} / \epsilon} \frac{F_{1}(a)}{F_{0}(a)} .
$$

Now it is possible to find $U_{w 1}$, which turns out to be now unique and differ from 


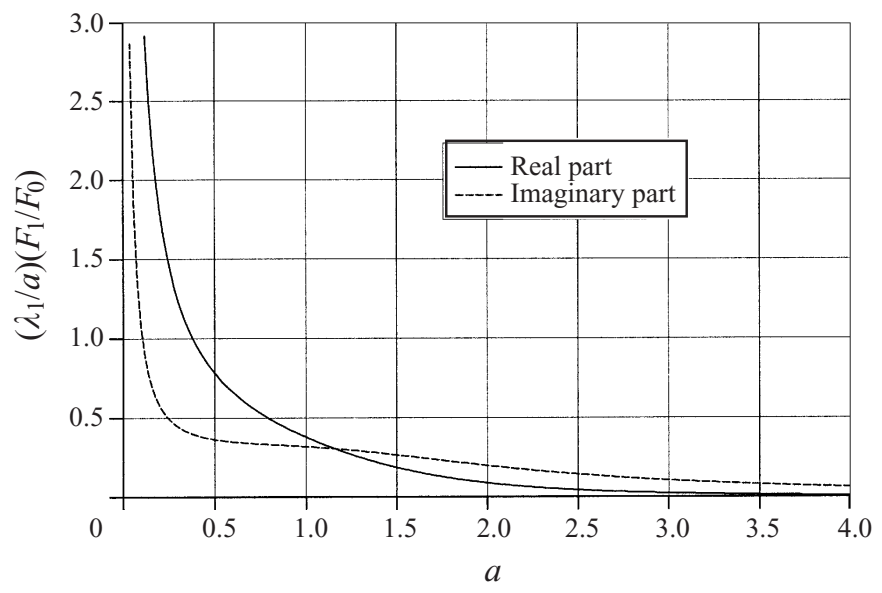

FIGURE 8. Dependence of $\left(\lambda_{1} / a\right)\left(F_{1} / F_{0}\right)$ upon $a$.

the zero value given by Glauert's solution with no suction by the amount

$$
U_{w 1}=2 \operatorname{Re}\left[-\frac{\lambda_{1}}{a} \mathrm{e}^{\left(\lambda_{1}-\lambda_{0}\right) \psi^{*} / \epsilon}\left(\frac{F_{1}(a)}{F_{0}(a)}\right)\right],
$$

where (6.5) for $C_{0}$ and $C_{1}$ was used. The factor 2 appears after adding the complex conjugate solution, corresponding to $n=-1$. This expression depends on the combination $\left(\lambda_{1} / a\right)\left(F_{1}(a) / F_{0}(a)\right)$ which is plotted in figure 8 as a function of $a$. In addition, the order of $U_{w 1}$ is determined by the factor $\mathrm{e}^{\left(\lambda_{1}-\lambda_{0}\right) \psi^{*} / \epsilon}$, which is exponentially small for all $a \geqslant 0$.

It is not clear whether taking into account the higher-order power terms in the expansion as $R e \rightarrow \infty$ will affect (6.13), but at least we have demonstrated that $U_{w 1}=o(1)$ without use of the invariant condition of vorticity necessarily changing sign on a closed contour surrounding the body. This result explains in more detail the mechanism of the loss of uniqueness of the standard asymptotic solution revealed in the previous sections. The usual approach to resolving difficulties associated with nonuniqueness of a certain term of the expansion consists in the analysis of the higherorder terms. Then the unique solution is obtained from the solvability condition of the problem for some higher term of the expansion. In the problem considered in the present paper, the incorrect choice of $U_{w}$ would lead to discrepancies so small that they would not manifest themselves in any term of the expansion proportional to a power of $R e$. Namely, if $U_{w}$ is not correct then there is an exponentially small non-zero vorticity flux in the wake. On the characteristic scale of any term of the expansion in the boundary layer this flux is zero. However, the characteristic scale of the vorticity in the mixing layer is also small and there this non-zero flux has already included manifested itself in the main term of the expansion. This was in fact used to determine the unique value of $U_{w 1}$.

Note also that even outside the boundary layer the expansion for the velocity contains power terms, which we did not calculate but which are much greater than the exponentially small term corresponding to the vorticity term found in this section. However, for the vorticity itself the term found is the main term and it happens to be important. 


\section{Conclusion}

The results obtained give strong evidence in favour of the following conclusions.

The high-Reynolds-number asymptotics of the steady flow past a quickly rotating cylinder with suction is unique.

If the flow at infinity is not uniform but has a small uniform shear or, equivalently, a small constant vorticity then, naturally, the velocity circulation around the cylinder will depend not only on the rotation velocity but also on this vorticity. It turns out that to change the circulation by a quantity of order unity it is sufficient to change the value of the vorticity at infinity by a quantity which is exponentially small as the Reynolds number tends to infinity. For the particular problem considered in the present paper the method of matched asymptotic expansion cannot distinguish exponentially small quantities and, as a result, solutions obtained by this method seem to be not unique.

Sometimes the unique solution can be determined by the requirement that it should satisfy a certain invariant condition which can be derived from the full Navier-Stokes equations for arbitrary Reynolds number and which must be valid in the asymptotic limit. In general explicit analysis of the exponentially small terms is necessary. This type of analysis was performed for the vorticity distribution outside the boundary layer.

It is interesting to note an important role of the velocity at infinity in this problem. For a zero value of the velocity the solution is known to be not unique. In all cases in this paper where the uniqueness was demonstrated the velocity at infinity was not zero. Indeed, for suction of order one we used the condition of vorticity necessarily changing sign on the contour enclosing the body, but this condition itself is valid only in the case of non-zero velocity at infinity. For suction of order $1 / \sqrt{R e}$ and $1 / R e$ the existence of an outermost closed streamline was explicitly used, which again requires non-zero velocity at infinity.

The results obtained have certain methodological implications. Often, when a certain term of an asymptotic expansion seems to be not unique, that means that a solvability condition of the problem for some higher term must be used to eliminate the non-uniqueness. This is, for example, the case for the famous Prandtl-Batchelor theorem, when the use of the invariant condition of zero flux through a closed contour (as in Batchelor 1956) is not the only way to determine a unique solution. However, in the problem considered in the present paper this higher-order term turns out to be exponentially small. In the cases when an invariant condition is not available for unique determination of the solution, methods of calculating exponentially small terms have to be developed.

The authors wish to thank the referees of this paper for their useful comments.

\section{REFERENCES}

BATCHELOR, G. K. 1956 On steady laminar flow with closed streamlines at large Reynolds number. J. Fluid Mech. 1, 177-190.

Chernyshenko, S. I. 1995 Asymptotics of steady axisymmetric flow of incompressible fluid past a bluff body at high Reynolds number. Izv. RAN, Mekh. Zhidk. i Gaza 1, 37-34 (in Russian).

Chernyshenko, S. I. 1998 Asymptotic theory of global separation. Appl. Mech. Rev. 9, 523-536.

Courant, R. 1962 Partial Differential Equations. Interscience.

EGOROV, I. V. 1986 Study of the flow past a circular cylinder with strong suction through its surface. Uchenye Zapiski TsAGI 3, 34-40 (in Russian). 
Glauert, M. B. 1957 The flow past a rapidly rotating circular cylinder. Proc. R. Lond. Soc. A 242, $108-115$.

Golubkin, V. N. \& SizYKh G. B. 1987 On certain general properties of plane flows of viscous fluid. Izv. Acad. Nauk SSSR, Mekh. Zhidk. i Gaza 3, 176-178 (in Russian).

Kuksin, S. B. 1997 Private communication.

NeIland, V. Ya. \& SycheV, V. V. 1966 Asymptotic solutions of the Navier-Stokes equations in regions with large local perturbations. Izv. Acad. Nauk SSSR, Mekh. Zhidk. i Gaza 4, 43-49 (in Russian).

Nikolaev, K. V. 1982 Separation appearance at the rotating cylinder in a uniform stream of incompressible fluid. Uchenye Zapiski TsAGI 6, 32-39 (in Russian).

Prandtl, L. W. \& Tietjens, O. G. 1936 Applied Hydro- and Aero-Mechanics. McGraw-Hill.

RosenheAD, L. 1963 Laminar Boundary Layers. Oxford University Press.

SQuire, H. B. 1956 Note on the motion inside a region of recirculation (cavity flow). J. R. Aero. Soc. 60, 203.

WooD, W. W. 1957 Boundary layers whose streamlines are closed. J. Fluid Mech. 2, 77-87. 